\title{
A deep intronic mutation in the $R B 1$ gene leads to intronic sequence exonisation
}

\author{
Catherine Dehainault ${ }^{1}$, Dorothée Michaux ${ }^{1}$, Sabine Pagès-Berhouet ${ }^{1}$, \\ Virginie Caux-Moncoutier ${ }^{1}$, François Doz ${ }^{2}$, Laurence Desjardins ${ }^{3}$, Jérôme Couturier ${ }^{1,4}$, \\ Philippe Parent ${ }^{5}$, Dominique Stoppa-Lyonnet ${ }^{1,4}$, Marion Gauthier-Villars ${ }^{1}$ \\ and Claude Houdayer*,1,6
}

\begin{abstract}
${ }^{1}$ Service de Génétique Oncologique, Institut Curie, Paris, France; ${ }^{2}$ Service d'Oncologie Pédiatrique, Institut Curie et Faculté de Médecine Paris René Descartes, Paris, France; ${ }^{3}$ Service d'Ophtalmologie, Institut Curie, Paris, France; ${ }^{4}$ INSERM U509, Pathologie Moléculaire des Cancers, Institut Curie, Paris, France; ${ }^{5}$ Service de Génétique Médicale, CHU Brest, Hôpital Morvan, Brest, France; ${ }^{6}$ UMR INSERM 745 Université René Descartes Paris, France
\end{abstract}

Familial forms of retinoblastoma, an embryonic neoplasm of retinal origin, are caused by constitutional mutations of the $R B 1$ gene. In this paper, we describe a family with retinoblastoma affecting two brothers with no previous family history of cancer. Complete $R B 1$ mutational screening including point mutation and large rearrangement screening failed to demonstrate any mutation. The whole coding sequence was therefore investigated at the cDNA level, demonstrating a $103 \mathrm{bp}$ intronic insertion between exons 23 and 24, leading to subsequent frameshift and premature termination of translation. This intronic exonisation was caused by a deep intronic mutation in intron 23 generating a cryptic $3^{\prime}$ splice site. This is the first report of a deep intronic mutation in $R B 1$ and is a proof of concept that some undetected $R B 1$ mutations should be investigated at the cDNA level, particularly in hereditary forms of retinoblastoma.

European Journal of Human Genetics (2007) 15, 473-477. doi:10.1038/sj.ejhg.5201787; published online 14 February 2007

Keywords: retinoblastoma; RB1; deep intronic; mutation; exonisation; cDNA screening

\section{Introduction}

Retinoblastoma (RB) [MIM 180200] is an embryonic neoplasm of retinal origin with an incidence of 1 in 15000 . It almost always arises in early childhood and is caused by inactivation of both alleles of the RB1 gene, within chromosome bands $13 q 14.2 .^{1}$ In non-hereditary retinoblastoma, both mutations in $R B 1$ take place in a single retinal cell that develops into the tumour. In hereditary retinoblastoma, germline mutation of one allele is associated with predisposition to retinoblastoma. The germline mutation is either inherited from an affected parent (hereditary familial RB) or acquired during gameto-

*Correspondence: Dr C Houdayer, Service de Génétique Oncologique, Institut Curie, 75248 Paris Cedex 05, France.

Tel: + 331443246 98; Fax: + 331443245 09;

E-mail: claude.houdayer@curie.net

Received 21 November 2006; revised 30 December 2006; accepted 5 January 2007; published online 14 February 2007 genesis or gestation (hereditary de novo). All kinds of mutations are found in the RB1 mutational spectrum making $R B 1$ screening extremely challenging as the majority of the mutations are unique and spread over the entire coding sequence. ${ }^{2}$. The mutation detection rate is expected to be $100 \%$ in hereditary familial RB, but some of these cases appear to escape detection despite a wholecoding sequence analysis that is large rearrangement and point mutation screening. These undetected mutations may actually be deep intronic alterations that could impact on normal splicing and consequently be responsible for the patient's disease. ${ }^{3}$ Exon recognition is accomplished by the accumulated recognition of multiple weak signals, resulting in a network of interactions across exons as well as across introns. Therefore one can expect a number of deleterious mutations in exons or introns that disrupt or create auxiliary cis-elements and classical splice sites. ${ }^{4}$ 
Most of the RB1 splice mutations described to date affect canonical splice sites resulting in skipping of the neighbouring exon (retinoblastoma genetics, available at http:// rb1-lsdb.d-lohmann.de/). The creation of a cryptic splice site in the central part of a large intron has not been reported to date in $R B 1$. In this paper, we describe the identification and characterization of a deep intronic mutation in an RB family leading to the insertion in the transcript of an out-of-frame $103 \mathrm{bp}$ intronic sequence.

\section{Patients and methods \\ Patients}

Both patients belong to a family of Caucasian origin and were followed at the Institut Curie.

These patients were examined by an ophthalmologist, a paediatrician and a geneticist. $\mathrm{RB}$ was diagnosed at the age of 2 years in both patients (II-3 and II-4, Figure 1) on the basis of current ophthalmological and histological criteria. Patients II-3 and II-4 were diagnosed with bilateral RB and unilateral, unifocal $\mathrm{RB}$, respectively. Ocular fundus examination was normal in both parents (I-1 and I-2, Figure 1) and no family history of cancer was reported in the maternal and paternal branches. An individual written consent for molecular analysis was obtained from all sampled individuals or their legal guardians.

\section{Mutation analysis}

Routine genomic screening The patients were first screened using DNA extracted from a whole-blood sample collected on EDTA. The whole RB1 coding sequence with exon-intron boundaries was screened using a previously described strategy ${ }^{5}$ that combines denaturing high-performance liquid chromatography (DHPLC) and quantitative multiplex PCR of short fluorescent fragments (QMPSF) for point mutation and large rearrangement screening, respectively.

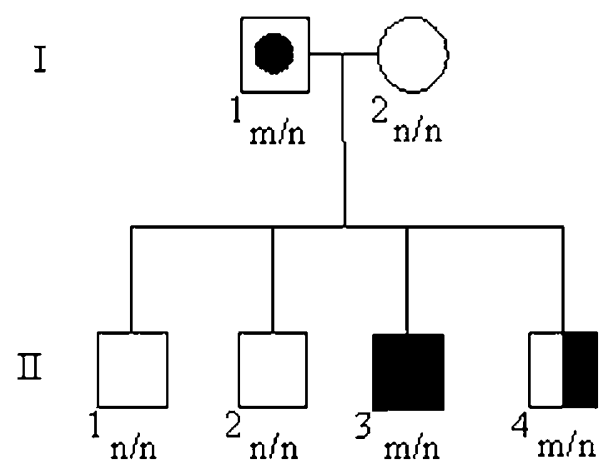

Figure 1 Family pedigree. Blackened symbol: Bilateral retinoblastoma; half-blackened symbol: unilateral retinoblastoma; dotted symbol: unaffected carrier. Genotype is provided for tested members as $\mathrm{m} / \mathrm{n}$ for heterozygous carriers and $\mathrm{n} / \mathrm{n}$ for homozygous wild-type.
cDNA sequencing Patient II-3 (Figure 1) and normal controls were further investigated at the cDNA level using RNA extracted from lymphoblastoid cell lines treated with and without puromycin. Puromycin treatment was used to inhibit nonsense mediated decay (NMD). ${ }^{6}$ RNA was reverse-transcribed ${ }^{5}$ and the whole $R B 1$ coding sequence was amplified in seven overlapping fragments (primer sequences available on request). Amplicons were purified and sequenced in both directions using the BigDye Terminator Cycle Sequencing V1.1 Ready Reaction kit (Applied Biosystems) with incorporation of the PCR oligonucleotides as extension primers, and following electrophoresis in an ABI PRISM 3130XL Genetic Analyzer with analysis using the Collection and Sequence Analysis software package (Applied Biosystems).

Transcript-specific analysis Following identification, the mutant transcript was specifically amplified using a dedicated primer containing a single $3^{\prime}$ locked nucleic acid base at the $3^{\prime}$ terminal position (AAAATGACTCCAAGAT CAAGT), referred to as LNA primer ${ }^{7}$ and sequenced as described above.

BLAST analysis The inserted exonic sequence was entered into BLAST (http://www.ncbi. nlm.nih.gov/BLAST/) and a search for exact matches was run in order to determine its location at the genomic level.

Characterization of the genomic mutation A genomic primer pair was designed to specifically amplify the exonised intronic sequence and its flanking regions (GCCAATCTGCTAAACAAAGCA, forward and AGCACTT GTGGTTGGATGTG, reverse). Amplicons were sequenced as described above.

Nucleotide position was numbered on the basis of the genomic sequence (GenBank accession number L11910.1) and according to recommended guidelines (available at http://www.emqn.org/emqn.php). We then searched for the mutation in all family members. All results were confirmed on a second blood sample.

Control group screening Absence of the genomic mutation was checked by direct sequencing on a panel of 96 control chromosomes derived from unrelated individuals belonging to breast/ovarian cancer families.

Splice site score predictions The genomic sequence environment of the mutation was analysed for $5^{\prime}$ and $3^{\prime}$ splice sites using Splice Site Prediction by Neural Network (NNSPLICE available at http://www.fruitfly.org/seq_tools/ splice.html), ${ }^{8}$ Splice Site Finder (SSF available at http:// violin.genet.sickkids.on.ca/ ali/splicesitefinder.html), Max EntScan (MES available at http://genes.mit.edu/burgelab/ maxent/Xmaxentscan_scoreseq.html). ${ }^{9}$ 
$6 \mathrm{~kb}$

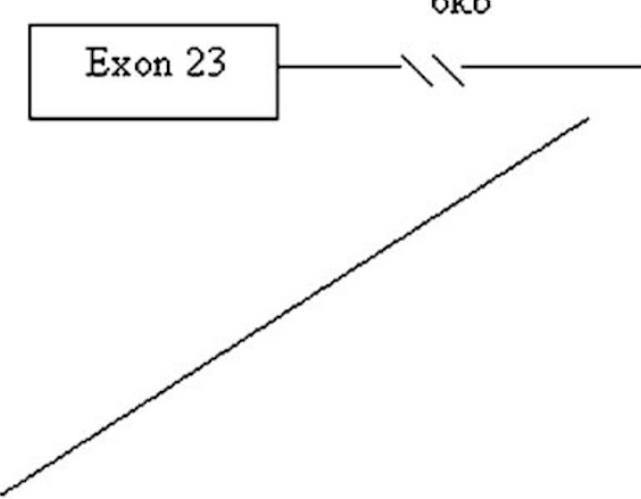

$2 \mathrm{~kb}$

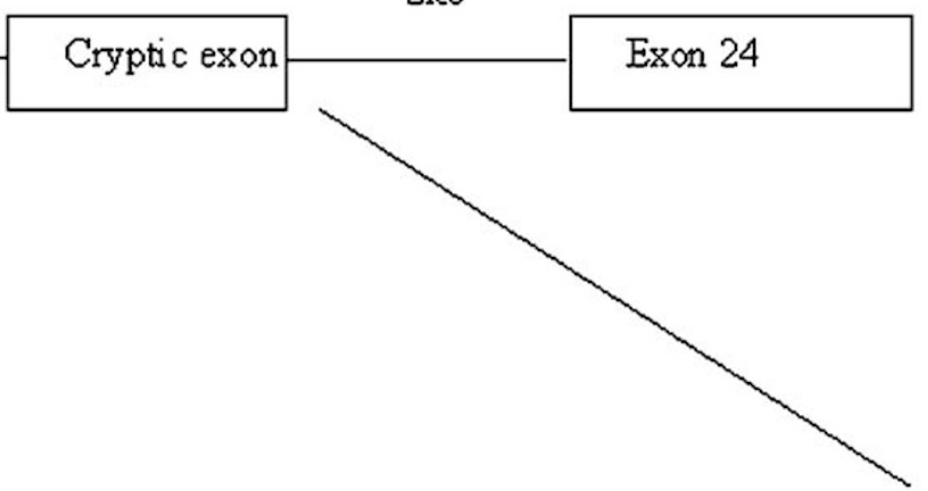

\section{aaatgtagattactgtcaattaagTCTTTAGAGGTCCATCCCTAATCCTGCTGGCGGCCTCTTTAC}

\section{CACCTCACCTTGGGCAGGTCTCTATCTGTACTTCACAAGGGTGCTGTGGATCAGG GAAATGatgagtatgaagctgtttaaattctcag}

b

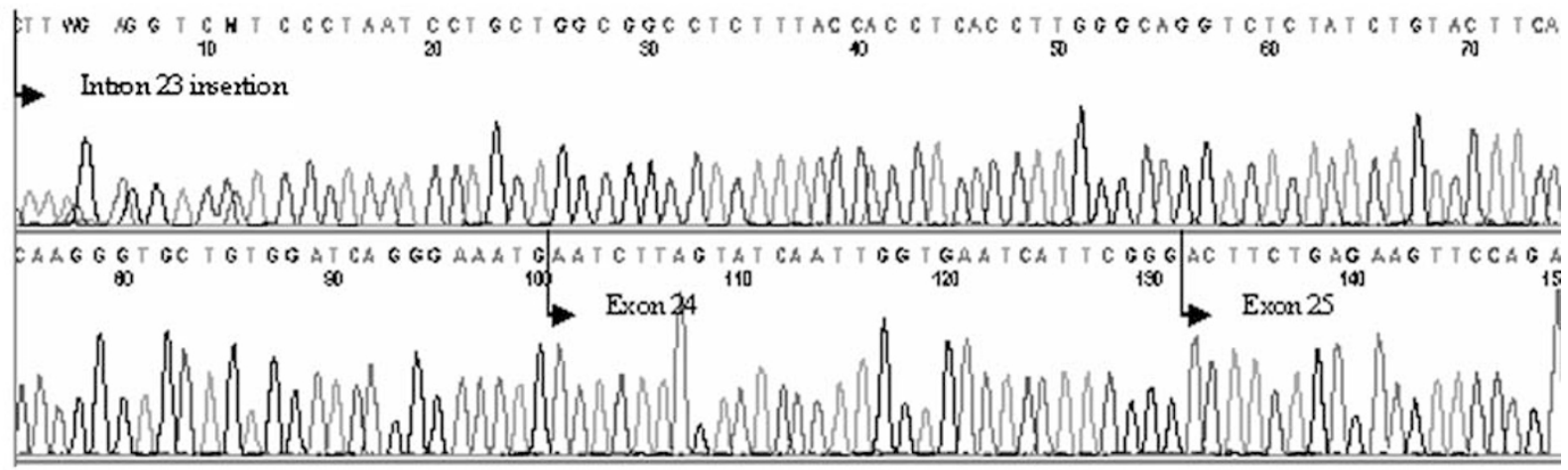

c

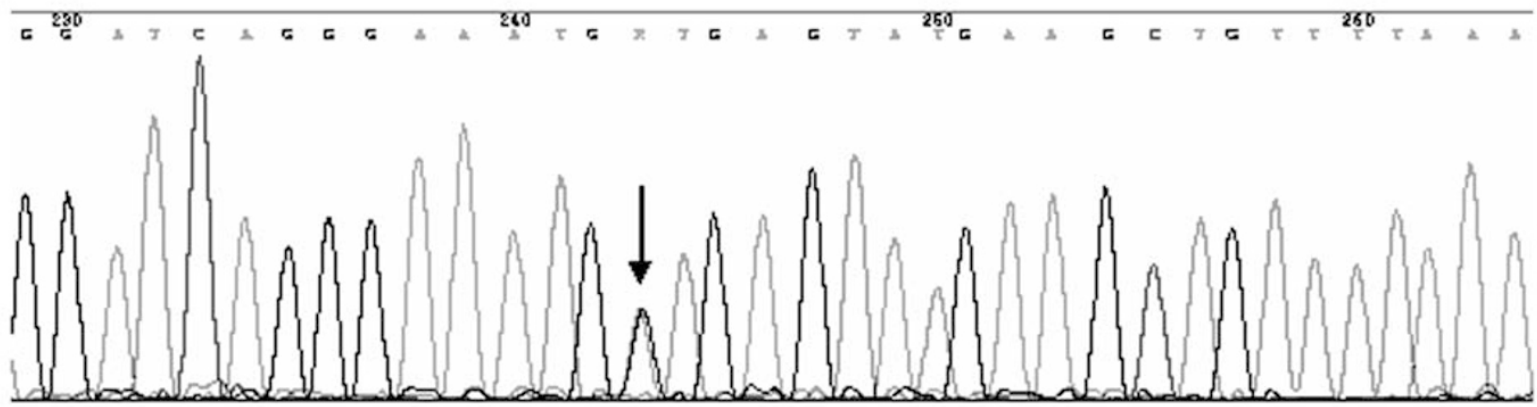

Figure 2 IVS23-1398A > G in patient II-3. (a) Genomic localization and sequence of the cryptic exon. The intronic exonised sequence is shown in capital letters, the IVS23-1398A > G mutation (indicated by an arrow) creates a $5^{\prime}$ cryptic splice site with a very high score that is $88.9,0.99$ and 10.13 using NNSPLICE, SSF and MES, respectively. The pre-existing 3 'splice site retains some homology with the consensus sequence (underlined). (b) Sequence of the mutant transcript following allele-specific amplification. The inserted sequence from intron 23 , exons 24 and 25 is indicated by arrows. (c) Characterization of the IVS23-1398A > G mutation in patient II-3. The mutation is indicated by an arrow. 
Presence of Exonic Splicing Enhancers (ESE) was checked using ESE Finder (available at http://rulai.cshl.edu/tools/ $\mathrm{ESE} /$ ) $^{10}$ and RESCUE-ESE (available at http://genes.mit.edu/ burgelab/rescue-ese/). ${ }^{11}$

\section{Results}

Mutation identification and characterization

Point mutation and large rearrangement screening on genomic DNA failed to demonstrate any mutation in either of the affected brothers. As an oncogenic RB1 mutation is expected in this hereditary familial form, we performed a complete transcript sequence analysis using RNA extracted from lymphoblastoid cell lines treated with and without puromycin. A 103-bp-insertion between exons 23 and 24 was found in cDNA originating from treated cell lines. Of note, this insertion was not detected in cDNA from untreated cells. Thorough analysis of the sequence data and further specific amplification of the mutant transcript followed by BLAST analysis showed that this insertion was part of $R B 1$ intron 23, encompassing nucleotides 168871 to 168973 (Figure 2a and $b$ ). To shed light on the mechanism involved in this intronic sequence exonisation, we sequenced this part of intron 23 plus the flanking regions, to detect a g.168974A $>$ G/IVS23-1398A $>$ G base substitution (Figure 2c). This mutation was found in the leucocyte DNA of both affected brothers and their father but was absent from our control panel of 96 chromosomes.

\section{Splice site prediction}

In silico analysis using NNSPLICE, SSF and MES demonstrated the creation of a $5^{\prime}$ cryptic splice site with a very high score that is 88.9, 0.99 and 10.13, respectively (Figure 2a). ESE finder found a large number of ESE throughout the insertion. On the other hand, RESCUE ESE found four ESEs, three of which were close to the cryptic $5^{\prime}$ splice site (data not shown).

\section{Discussion}

Two patients diagnosed with $\mathrm{RB}$ who were tested negative for point mutation and large rearrangements were investigated by thorough cDNA analysis. A deep intronic mutation leading to an intronic sequence exonisation was found (g.168974A>G/c.2490_2491ins103/p.Ile831SerfsX7). This mutation can be classified as deleterious since it creates an out-of-frame transcript with a subsequent premature stop codon.

Moreover, the mutation was found in the two affected brothers but not in a control panel of 96 chromosomes. Of note, the unaffected father (I-1, Figure 1) also carried this mutation. Although his parents were unavailable for testing, the absence of family history strongly suggests that the mutation occurred de novo in the father. Consequently, he probably carried the mutation in a mosaic state thereby explaining this lack of penetrance. ${ }^{12,13}$ On the other hand, his carrier offspring developed the disease since the oncogenic mutation was present in each of their cells. Assuming a mosaic state in the father, the observed phenotype is in line with expectations for a frameshift mutation (disease-eye-ratio $(\mathrm{DER})=1.5$, first generation carrier excluded). ${ }^{14}$ Alternatively, we could not formally exclude the possibility that the IVS23-1398A > G substitution acts as a low penetrance mutation since the relative proportions of aberrantly and correctly spliced transcripts may explain the reduced disease severity observed. ${ }^{15,16}$ However, why this would be the case in some family members and not in others remains unclear.

This is the first report of a deep intronic mutation in $R B 1$, added to several reports of intronic sequence exonisation for example in the $A T M,{ }^{17} C F T R,{ }^{3,18} D M D,{ }^{19,20}$ and $N F 1^{21}$ genes. In these cases, the intronic mutation activates a cryptic donor or acceptor splice site and splicing between this novel splice site and a pre-existing, normally silent, splice site leads to the inclusion of a cryptic exon. We observed a similar mechanism since in silico predictions showed that the IVS23-1398A > G mutation creates a highquality donor splice site. What is surprising in our case is that this cryptic donor site interacts with a poor quality pre-existing acceptor site (Figure 2a) although it retains some homology with the consensus sequence. ${ }^{22}$ This could be explained by: (i) co-activation of 'cryptic' ESEs since ESE Finder predicts a large number of ESE throughout the inserted sequence; (ii) failure of the matrix used for calculation, as accurate recognition of acceptor sites appears to be a challenging task. ${ }^{23}$

Overall, this report is a plea for cDNA screening when an expected mutation is not detected by classical approaches. As demonstrated here, this screening should be performed with cells treated with puromycin since an out-of-frame defect is most probably involved. Use of normal controls is also mandatory to avoid spurious interpretations. This should encourage clinical and molecular geneticists to complete their mutational screening in hereditary forms of retinoblastoma without mutation.

\section{Acknowledgements}

The authors thank I Eugene, S Miglierina and C Postel for helpful support during the study. This work was supported by a grant from Retinostop and Programme Incitatif et Coopératif 'Rétinoblastome', Institut Curie.

\section{References}

1 Friend SH, Bernards R, Rogelj S et al: A human DNA segment with properties of the gene that predisposes to retinoblastoma and osteosarcoma. Nature 1986; 323: 643-646.

2 Richter S, Vandezande K, Chen N et al: Sensitive and efficient detection of RB1 gene mutations enhances care for families with retinoblastoma. Am J Hum Genet 2003; 72: 253-269. 
3 Highsmith WE, Burch LH, Zhou Z et al: A novel mutation in the cystic fibrosis gene in patients with pulmonary disease but normal sweat chloride concentrations. N Engl J Med 1994; 331: 974-980.

4 Faustino NA, Cooper TA: Pre-mRNA splicing and human disease. Genes Dev 2003; 17: 419-437.

5 Houdayer C, Gauthier-Villars M, Lauge A et al: Comprehensive screening for constitutional RB1 mutations by DHPLC and QMPSF. Hum Mutat 2004; 23: 193-202.

6 Andreutti-Zaugg C, Scott RJ, Iggo R: Inhibition of nonsensemediated messenger RNA decay in clinical samples facilitates detection of human MSH2 mutations with an in vivo fusion protein assay and conventional techniques. Cancer Res 1997; 57: 3288-3293.

7 Latorra D, Campbell K, Wolter A, Hurley JM: Enhanced allelespecific PCR discrimination in SNP genotyping using $3^{\prime}$ locked nucleic acid (LNA) primers. Hum Mutat 2003; 22: 79-85.

8 Reese MG, Eeckman FH, Kulp D, Haussler D: Improved splice site detection in Genie. J Comput Biol 1997; 4: 311-323.

9 Yeo G, Burge CB: Maximum entropy modeling of short sequence motifs with applications to RNA splicing signals. J Comput Biol 2004; 11: 377-394.

10 Cartegni L, Wang J, Zhu Z, Zhang MQ Krainer AR: ESEfinder: a web resource to identify exonic splicing enhancers. Nucleic Acids Res 2003; 31: 3568-3571.

11 Fairbrother WG, Yeh RF, Sharp PA, Burge CB: Predictive identification of exonic splicing enhancers in human genes. Science 2002; 297: 1007-1013.

12 Carlson EA, Desnick RJ: Mutational mosaicism and genetic counseling in retinoblastoma. Am J Med Genet 1979; 4: $365-381$

13 Sippel KC, Fraioli RE, Smith GD et al: Frequency of somatic and germ-line mosaicism in retinoblastoma: implications for genetic counseling. Am J Hum Genet 1998; 62: 610-619.
14 Lohmann DR, Brandt B, Hopping W, Passarge E, Horsthemke B: Distinct RB1 gene mutations with low penetrance in hereditary retinoblastoma. Hum Genet 1994; 94: 349-354.

15 Lohmann DR, Gallie BL: Retinoblastoma: revisiting the model prototype of inherited cancer. Am J Med Genet C Semin Med Genet 2004; 129: 23-28.

16 Tuffery-Giraud S, Saquet C, Thorel D et al: Mutation spectrum leading to an attenuated phenotype in dystrophinopathies. Eur J Hum Genet 2005; 13: 1254-1260.

17 Pagani F, Buratti E, Stuani C, Bendix R, Dork T, Baralle FE: A new type of mutation causes a splicing defect in ATM. Nat Genet 2002; 30: $426-429$.

18 Chillon M, Dork T, Casals T et al: A novel donor splice site in intron 11 of the CFTR gene, created by mutation $1811+1.6 \mathrm{kbA}-$ $>\mathrm{G}$, produces a new exon: high frequency in Spanish cystic fibrosis chromosomes and association with severe phenotype. Am J Hum Genet 1995; 56: 623-629.

19 Beroud C, Carrie A, Beldjord C et al: Dystrophinopathy caused by mid-intronic substitutions activating cryptic exons in the DMD gene. Neuromuscul Disord 2004; 14: 10-18.

20 Tuffery-Giraud S, Saquet C, Chambert S, Claustres M: Pseudoexon activation in the DMD gene as a novel mechanism for Becker muscular dystrophy. Hum Mutat 2003; 21: 608-614.

21 Raponi M, Upadhyaya M, Baralle D: Functional splicing assay shows a pathogenic intronic mutation in neurofibromatosis type 1 (NF1) due to intronic sequence exonization. Hum Mutat 2006; 27: 294-295.

22 Cartegni L, Chew SL, Krainer AR: Listening to silence and understanding nonsense: exonic mutations that affect splicing. Nat Rev Genet 2002; 3: 285-298.

23 Vorechovsky I: Aberrant $3^{\prime}$ splice sites in human disease genes: mutation pattern, nucleotide structure and comparison of computational tools that predict their utilization. Nucleic Acids Res 2006; 34: 4630-4641. 